J. Orn. 125, 1984: S. $75-79$

\title{
Der Brutraum beeinflußt Gelegegröße und Fortpflanzungserfolg beim Star (Sturnus vulgaris)
}

\section{Von Fritz Trillmich und Hans Hudde}

LöHRL $(1973,1980)$ wies in eleganten Versuchsreihen nach, daß Kohlmeisen (Parus major) in größeren Nisthöhlen größere Gelege und höheren Bruterfolg haben als in kleinen. Er konnte (1970) außerdem zeigen, daß Kohlmeisen größere Höhlen deutlich bevorzugen. Ein solcher Einfluß der Nistkastengröße auf die Gelegegröße („Löhrl-Ef$\mathrm{fekt}^{\text {" }}$ ) ist inzwischen auch für weitere Arten nachgewiesen worden. KARLSSON \& NILSson (1977) zeigten, daß der Löhrl-Effekt bei Sumpf- ( $P$. palustris) und Weidenmeise ( $P$. montanus; nach LUDESCHER 1973) am deutlichsten, bei Kohlmeise und Trauerschnäpper (Ficedula bypoleuca) schwächer ist, während er beim Star (Sturnus vulgaris) im Bereich der getesteten Nistkastengrößen nicht nachweisbar war.

\section{Material und Methode}

Die Untersuchungen wurden 1979-1983 in vier Gebieten durchgeführt. Gebiet 1 liegt am Altwasser der Ammer bei Fischen (Lkrs. Weilheim-Schongau, Bayern), Gebiet 2 ist ein Teil des Parkes der Villa Hügel in Essen, Gebiet 3 ein Wald bei Isenbüttel (Wolfsburg) und Gebiet 4 ein Wald bei Harthausen (Speyer). Die Nistkästen waren in Waldrandnähe aufgehängt. In unmittelbarer Umgebung befanden sich Wiesen und in den Gebieten 1, 3 und 4 auch Äcker.

Drei Typen von Nistkästen wurden verwendet: Enge, zylindrische Holzbetonkästen (Firma Schwegler) mit $143 \mathrm{~cm}^{2}$ Grundfläche (= Holzbetonkästen), enge Holzkästen mit kleiner quadratischer Grundfläche von $154 \mathrm{~cm}^{2}$ (= kleine Holzkästen) und weite Holzkästen (= große Kästen) mit quadratischer Grundfläche von $361 \mathrm{~cm}^{2} .1979$ wurden nur Holzbetonkästen ausgehängt, 1980 Holzbetonkästen und große Kästen gemischt. Ab 1981 wurden in den Gebieten 1, 3 und 4 nur große Kästen geboten. In Gebiet 2 wurden 1981 und 1982 alle 3 Nistkastentypen gemischt und 1983 ausschließlich kleine Holzkästen geboten. Die Nistkästen waren immer in einem Mindestabstand von $5 \mathrm{~m}$ in $3-4 \mathrm{~m}$ Höhe aufgehängt.

Die Gelegegröße wurde durch mindestens 2 Kontrollen während der Lege- und Bebrütungszeit bestimmt. Jungvögel wurden am 10. bis 15. Lebenstag im Rahmen einer Populationsstudie beringt. Die Zahl ausgeflogener Junger bestimmten wir aus der Zahl der Jungen bei der Beringung abzüglich tot gefundener Junger bei einer Nistkastenkontrolle kurz nach dem Ausfliegen.

Den Mitarbeitern bei dieser Untersuchung D. Hoffmann, H. Schemmel und J. Steiger, sowie C. Kasche und L. Bats gebührt ganz herzlicher Dank für ihre Freilandarbeit. Dr. E. Gwinner, J. Lamprecht, H. Löhrl, C. Rechten, H.-U. Reyer, U. SeibT und W. Wickler lasen das Manuskript kritisch und halfen mit Vorschlägen zu seiner Verbesserung. B. KNAUER zeichnete die Abbildung.

\section{Ergebnisse}

1. Präferenz für große Nistkästen

1980 wurden in den Versuchsgebieten große Holzkästen und kleine Holzbetonkästen gemischt geboten. Wie Tab. 1 zeigt, zogen die Stare in der Erstbrut die großen 
Kästen eindeutig den Holzbetonkästen vor. Um sicherzustellen, daß es sich hierbei um eine Präferenz für große Kästen und nicht um eine für Holz gegenüber Holzbeton handelte, wurden 1981 und 1982 kleine und große Holzkästen im Gebiet 2 gleichzeitig angeboten. Auch in dieser Wahlsituation zogen die Stare die großen Kästen signifikant vor (Tab. 1). Diese Bevorzugung bestand, obwohl die Stare in große Kästen wesentlich mehr Nistmaterial eintrugen als in kleine, also mehr Zeit und Energie für den Nestbau in großen Kästen aufwenden mußten.

Tab. 1. Präferenz der Stare für große Nistkästen gemessen an der Benutzung der Kästen in der Erstbrut. 1980 wurden in allen 4 Gebieten große Holzkästen und kleine Holzbetonkästen und 1981/82 in Gebiet 2 große und kleine Holzkästen gleichzeitig angeboten. - Preference of Starlings for large nest boxes as measured by occupancy in first broods. In 1980 large wooden and small wood-concrete nestboxes were given simultaneously in all 4 study areas. In 1981/82 large and small wooden nestboxes were presented simultaneously in study area 2.

\begin{tabular}{llcccccc}
\hline $\begin{array}{l}\text { Kastentypen } \\
\text { Groß }\end{array}$ & klein & Große & Kleine & Große & Kleine & Große Kleine \\
\hline Holz & Holzbeton & 50 & 94 & 45 & 27 & 90 & 29 \\
Holz & Holz & 46 & 28 & 27 & 3 & 57 & 11 \\
\hline
\end{tabular}

1) Die Unterschiede sind in beiden Fällen signifikant (Chiquadrat-Test; $p<0.01$ ).

2. Unterschiede der Gelegegröße in Abhängigkeit von der Brutraumgröße

Entsprechend den Ergebnissen LöHRL's (1973, 1980) fanden sich auch beim Star in großen Kästen größere Gelege (Abb.). Im Mittel legten die Stare in der Erstbrut 4,3 Eier in Holzbetonkästen $(n=19)$ und ebenfalls 4,3 in kleine Holzkästen $(n=11)$, aber

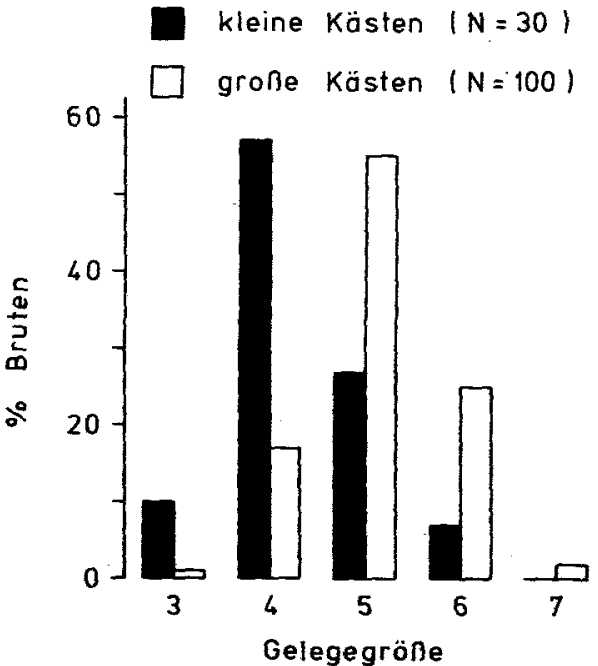

Die Verteilung der Gelegegröße in großen und kleinen Nistkästen. - Distribution of clutch sizes in large and small nestboxes. 
5,1 Eier in große Holzkästen $(n=177)$. Die Verteilungen der Gelegegrößen aus Holzbetonkästen und kleinen Holzkästen waren nicht unterscheidbar (Chiquadrat-Test und Mann-Whitney U-Test, $\mathrm{p}>0.5$ ). Diese beiden Kastentypen werden daher im folgenden gemeinsam als „kleine Kästen“ bezeichnet. In kleinen Kästen waren Gelege mit 4 Eiern am häufigsten, Gelege mit 6 Eiern recht selten. In großen Kästen waren 5 Eier am häufigsten, und Gelege mit 6 Eiern stellten $25 \%$ der Verteilung (Abb.). Die Verteilungen und Mittelwerte der Gelegegrößen in kleinen und großen Kästen sind signifikant verschieden (Chiquadrat-Test, $\mathrm{p}<0.001 ;$ Mann-Witney U-Test, $\mathrm{p}<0.0001$ ).

\section{Bruterfolg in großen und kleinen Nistkästen}

Stare verloren in kleinen Nistkästen mehr Bruten als in großen (Tab. 2). Dabei sind Bruten nicht berücksichtigt, die infolge unserer Störung, z. B. durch den Fang des Weibchens an der Nisthöhle, aufgegeben wurden.

Tab. 2. Brutverluste in der Erstbrut in großen und kleinen Nistkästen. Die Verlustraten sind signifikant verschieden (Chiquadrat-Test, $\mathrm{p}<0.02$ ). - Losses of first broods in large and small nestboxes. Percentage losses are significantly different.

\begin{tabular}{lrrr} 
& & \multicolumn{2}{c}{ Totalverluste } \\
& $\mathrm{n}$ & Vollgelege bis Schlupf & Schlupf bis Ausfliegen \\
\hline Große Kästen & 106 & $7 \%$ & $9 \%$ \\
Kleine Kästen & 30 & $23 \%$ & $21 \%$ \\
\hline
\end{tabular}

Für erfolgreiche Bruten waren in der Erstbrut Schlüpferfolg und Ausflugerfolg aus großen und kleinen Kästen nicht signifikant verschieden (Tab. 3). Stare, die in großen Kästen brüten, können also höheren Bruterfolg erwarten als Stare, die in kleinen Kästen brüten, weil sie in den größeren Kästen signifikant größere Gelege produzieren, die dann unter geringeren Verlusten aufgezogen werden.

Tab. 3. Bruterfolg in großen und kleinen Nistkästen. Totalverluste sind ausgeschlossen worden. - Breeding success in large and small nestboxes. Losses of total broods are excluded.

\begin{tabular}{lcccccc} 
& & \multicolumn{2}{c}{ Schlüpferfolg. } & Ausflugserfolg & \\
& Eizahl & $\mathrm{n}$ & Junge & $\left.0^{1}\right)$ & Junge & $\left.\% 0^{2}\right)$ \\
\hline \multirow{3}{*}{ Große } & 4 & 20 & 3.5 & 87 & 2.4 & 70 \\
Kästen & 5 & 54 & 4.3 & 87 & 3.3 & 77 \\
& 6 & 28 & 5.2 & 86 & 3.5 & 69 \\
& 7 & 3 & 6.0 & 86 & 5.0 & 83 \\
\hline \multirow{2}{*}{ Kleine } & 4 & 17 & 3.5 & 88 & 1.6 & 45 \\
Kästen & 5 & 8 & 4.0 & 80 & 3.3 & 80 \\
\hline
\end{tabular}

1) bezogen auf die Zahi der Eier im Vollgelege.

2) bezogen auf die Zahl geschlüpfter Junger. 


\section{Diskussion}

Die Brutraumgröße beeinflußt beim Star die Zahl der Eier und damit den Fortpflanzungserfolg. Dieser Befund steht im Widerspruch zu den Angaben KarLssons \& NiLssons (1977). Leider gaben die Autoren nur die Regressionsgleichungen für die Eizahl in Abhängigkeit von der Brutraumgröße und keinerlei Originaldaten. Daher ist ein genauer Vergleich mit unseren Daten nicht möglich. Allerdings prüften KarLsson \& Nilsson (1977) den Einfluß der Nistkastengröße nur über einen engen Bereich von Nistkastengrundflächen $\left(120-225 \mathrm{~cm}^{2}\right)$. Möglicherweise wird innerhalb dieses engeren Bereiches von relativ kleinen Nistkastengrößen der Löhrl-Effekt nicht.so deutlich.

Die Verringerung der Eizahl in kleinen Nisthöhlen könnte in Anpassung daran entstanden sein, daß die Temperaturbedingungen nach dem Schlüpfen der Jungen kritisch werden. Mertens (1977) wies für Kohlmeisen nach, daß die Gefahr der Überhitzung im Nistkasten weit größer ist als die der Unterkühlung. Vorversuche mit den von uns verwendeten Kästen haben ergeben, daß bei Wärmeeinstrahlung die Temperatur in kleinen Kästen höher ansteigt und daß nach Ende der Wärmeeinstrahlung die Abkühlung langsamer erfolgt als in großen Kästen. Dies liegt vermutlich an einer geringeren Luftzirkulation in den kleinen Kästen. Verringerte Zirkulation behindert aber auch die Ableitung der von größeren Jungvögeln selbst gebildeten Wärme. Überhitzung kann am besten vermieden werden, wenn die Jungvögel voneinander abrücken und durch vergrößerte Konvektion ihren Wärmeverlust erhöhen können. Diese Möglichkeit besteht in einem kleinen Nistkasten nur sehr begrenzt: bei Bruten mit 5 Jungen sitzen schon zehntägige Starenjunge mehr auf- als nebeneinander. Dann können die Elternvögel auch kaum mehr den Kot der Jungen entfernen. Die hygienischen Bedingungen sind daher in kleinen Kästen deutlich schlechter.

Die Gelegereduktion in kleinen Kästen (oder die Vergrößerung in großen) zeigt, daß Stare in der Lage sind, die Eizahl an den Brutraum anzupassen. Diese Anpassung ist vermutlich unter dem Selektionsdruck unterschiedlicher Nestlingsmortalität in verschieden großen Naturhöhlen entstanden und stellt einen unabhängig vom Nahrungsangebot (LACK 1968) wirkenden Faktor dar, der die Eizahl mitbestimmt (LòHrL 1980). Staren- $q$ inspizieren Bruthöhlen für einige Wochen, ehe sie mit der Eiablage beginnen. Falls sie of auch danach wählen, ob sie eine geeignete Höhle anbieten, könnte die Brutraumgröße eine wichtige Rolle im Rahmen der sexuellen Selektion spielen (SEARCY 1982).

Ludescher (1973) hat gezeigt, daß Sumpfmeisen und Weidenmeisen mit großen Höhlen größere Gelege produzieren. Dies ist anscheinend auch beim Buntspecht (Picoides major) der Fall. Gelege dieser Art haben im Mittel etwa 5,5 Eier; in kleinen Höhlen von nur $9 \times 9 \mathrm{~cm}$ Bodenfläche betrug der Mittelwert aber nur 3,4 Eier (GLuTz voN Blotzheim \& BAUER 1980, S. 1011). Brutraumgröße kann also auch bei Arten, die ihre Nisthöhlen selber herstellen, sehr variieren und die Eizahl beeinflussen. 


\section{Zusammenfassung}

Stare zogen große Nistkästen ( $361 \mathrm{~cm}^{2}$ Bodenfläche) kleinen $\left(143 \mathrm{~cm}^{2}\right.$ oder $154 \mathrm{~cm}^{2}$ Bodenfläche) vor. Gelege in großen Kästen hatten im Mittel der Erstbruten 5,1 Eier, Gelege in kleinen Kästen nur 4,3 Eier. Verluste der gesamten Brut waren in kleinen Kästen signifikant häufiger als in großen. Der Erfolg von Bruten, in denen überhaupt Junge bis zum Ausfliegen kamen, war in groBen und kleinen Kästen nicht signifikant verschieden. Die Gelegereduktion in kleinen Kästen stellt anscheinend eine Anpassung an erhöhte Nestlingsmortalität in kleinen Höhlen dar. Die Höhlengröße könnte beim Star eine wichtige Rolle im Rahmen der sexuellen Selektion spielen.

\section{Summary}

Nestbox area influences clutch size and reproductive success in the Starling (Sturnus vulgaris)

Starlings prefered large nestboxes (bottom area $361 \mathrm{~cm}^{2}$ ) over small ones (bottom area $143 \mathrm{~cm}^{2}$ or $154 \mathrm{~cm}^{2}$ ). Clutch size of first broods averaged 5.1 eggs in large nestboxes and only 4.3 eggs in small ones. Total brood loss occurred significantly more frequently in small than in large nestboxes. Brood success of productive broods was about the same in large and small nestboxes. Clutch size reduction in small nestboxes appears to prevent high nestling mortality in small nest cavities. In the Starling, size of nest holes may play an important role in the selection of males by females.

\section{Literatur}

Glutz v. Blotzheim, U. N., \& Bauer, K. M. (1980): Handbuch der Vögel Mitteleuropas. Bd. 9. Akademische Verlagsgesellschaft, Wiesbaden. - Karlsson, J., \& S. G. NiLsson (1977): The influence of nest-box area on clutch size in some hole-nesting passerines. Ibis 119:207-211. LACK, D. (1968): Adaptations for breeding in birds. Methuen, London. $\bullet$ LöHRL, H. (1970): Unterschiedliche Bruthöhlenansprüche von Meisenarten und Kleiber als Beitrag zum Nischenproblem. Verh. Deutsch. Zool. Ges. 64. Tagg.: 314-317. - Ders. (1973): Einfluß der Brutraumfläche auf die Gelegegröße der Kohlmeise (Parus major). J. Orn. 114: 339-347. - Ders. (1980): Weitere Versuche zur Frage „Brutraum und Gelegegröße“ bei der Kohlmeise, Parus major. J. Orn. 121: 403-405. Ludescher, F.-B. (1973): Sumpfmeise (Parus p. palustris L.) und Weidenmeise (P. montanus salicarius $\mathrm{Br}$.) als sympatrische Zwillingsarten. J. Orn. 114:3-56. - Mertens, J. A. L. (1977): Thermal conditions for successful breeding in Great Tits (Parus major L.). Oecologia 28: 1-29. SEARCY, W. A. (1982): The evolutionary effects of mate selection. Ann. Rev. Ecol. Syst. 13: $57-85$.

Anschrift der Verfasser: (F. T.) Max-Planck-Institut für Verhaltensphysiologie, Abt. Wickler, 8131 Seewiesen; (H. H.) Rüstermark 2, 4300 Essen-Stadtwald. 